\title{
Towards Intelligent Assembly and Manufacturing Environment - Modular ICT Support for Holonic Manufacturing System
}

\author{
Minna Lanz, Eeva Järvenpää, Pasi Luostarinen, \\ Fernando Garcia, and Reijo Tuokko \\ Tampere University of Technology (TTY-säätiö), P.O. Box 589, 33101 Tampere, Finland \\ \{minna.lanz, eeva.jarvenpaa, pasi.luostarinen, \\ fernando.garcia, reijo.tuokko\} atut.fi
}

\begin{abstract}
This paper discusses the possibilities of a modular and more transparent knowledge management concept for a holonic assembly and manufacturing environment that enhances representation of past and present status of the production system thus allowing autonomous reasoning to be applied. The modular ICT approach is developed for a holonic manufacturing system (HMS). The main characteristics of the system are that it is an open system and has characteristics of a complex system. The system itself is an adaptive system, where different services are needed depending on theevolution of the production system itself. The research approach represented here aims to enhance knowledge management and process control by facilitating the move from technology based solutions to conpgurable systems and processes where the digital models and modular knowledge management systems can be conPgured based on the need - not based on the closed legacy systems and Pxed process planning. The principles of modularization such as functionalities and interfaces, are used to deal with interactions of the full system.
\end{abstract}

Keywords: knowledge management, ontology, holonic manufacturing system, autonomous system, modular system.

\section{Introduction}

The operation and business environment for manufacturing industry changes rapidly. The adaptation is expected in design of products and processes and in realization of processes. Manufacturing industry can gain operational flexibility by rapid reaction and adaptation to these changes. In order to achieve the needed level of adaptability a company must be able to learn. The learning is achieved via gaining and understanding the feedback of the change: its quantity and direction. However, in order to gain and understand the feedback a company must be able to compare the past status to the new status of operations. Unfortunately, the knowledge of neither the past nor the present is in computer interpretable and comparable form. Thus the achieved and/or imagined flexibility is slightly above non-existent.

Manufacturing after all is the backbone of each and every society, and in order for society to sustain in long run the manufacturing has to be sustainable as well. From 
other point of view the assembly and manufacturing systems are shifting from being to becoming. This means that as the intelligence and cooperativeness advances the system will become a society where the rules, possibilities and constrains of a society as we know it will also apply.

While it is understood and accepted that the production systems are natural systems of a kind and those need to adapt to the changes demanded by the business domain. It is also undertood that the adaptive systems are also changing over their life-cycles. However, less notice is paid to the ICT systems that are supposed to be supporting this evolvable business and manufacturing domain. In order to support ever changing environment the ICT must also be adaptive system, where the tools follow their own life-cycle phases in regards to the changes in technology and business domains.

This paper introduces a concept for modular ICT architecture that supports dynamic and adaptive holonic manufacturing system by allowing new services to be implemented into the whole system during and depending its life-cycle status. The modular ICT approach is seen as one possible solution for capturing content and context information in different levels of abstraction and perhaps in future support real learning factories.

\section{State of the Art}

\subsection{Data and Information Modeling}

Starting from the ongoing research there can be found an increasing trend in different domain contexts on using emerging technologies such as ontologies, semantics and semantic web (Web 2.0), to support the collaborations and interoperability needs [4]. In recent years there have been a lot of activities in the field of modeling domain and upper ontologies for manufacturing. Lohse [11] defined the connection between processes and resources for modular assembly systems as a result for the FP6 EUPASS project. FP6 PABADIS'PROMISE [13] project resulted a manufacturing ontology (P2 ontology) and reference architecture focusing on factory floor control. Borgo et al. [1] developed an ADACOR ontology for distributed holon-based manufacturing focusing on processes and system interaction descriptions. ADACOR was later extended with an upper ontology (DOLCE). Research done in FP6 IP-PiSA project resulted an ontology, Core Ontology, for connecting product, process and system domains under one reference model [9]. The main goals these approaches generally try to achieve are: improved overall access to domain knowledge and additional information of a concept.

\subsection{Dynamic and Evolving Assembly Systems}

Dynamic and Evolving Assembly and Production systems generally target to the systems where change is frequent and typically characterized by low production volumes with high variety of products.

Onori [12] introduced the concept for Evolvable Production Systems [EPS] already in 2002. Since then Ribeiro et al. [14] have continued developding the concept within the area of complex systems. The main characteristics of the EPS is that the system is 
seen as evolving and adapting system thus having characteristics of an emerging system.

Likewise the PABADIS'PROMISE project provided a generic solution to the vertical interoperability problem from the enterprise level to the manufacturing level focusing on factory control and production scheduling in a multi-agent environment. However, in their approach the product data conversions were performed semiautomatically, while the production planning was agent-based [13].

Borgo et al. [1] utilized the ADACOR ontology in their HMS environment. The system considered entities performing the manufacturing scheduling and control operations. Along the domain description the ontology provided a set of rules and restrictions for different types of processes.

\section{Modular ICT for Dynamic and Evolvable Systems}

Since there does not exist a common knowledge representation that would unite different domain representations there is two research options that are in use. One of the solutions intended to for the knowledge representation is mapping between different representations, such as semantic-based XML schema matching by Kim et. al. [6]. This approach supports the existence of multiple different representations while advanced reasoning and mapping technologies are used for achieving information mapping from one model to another.

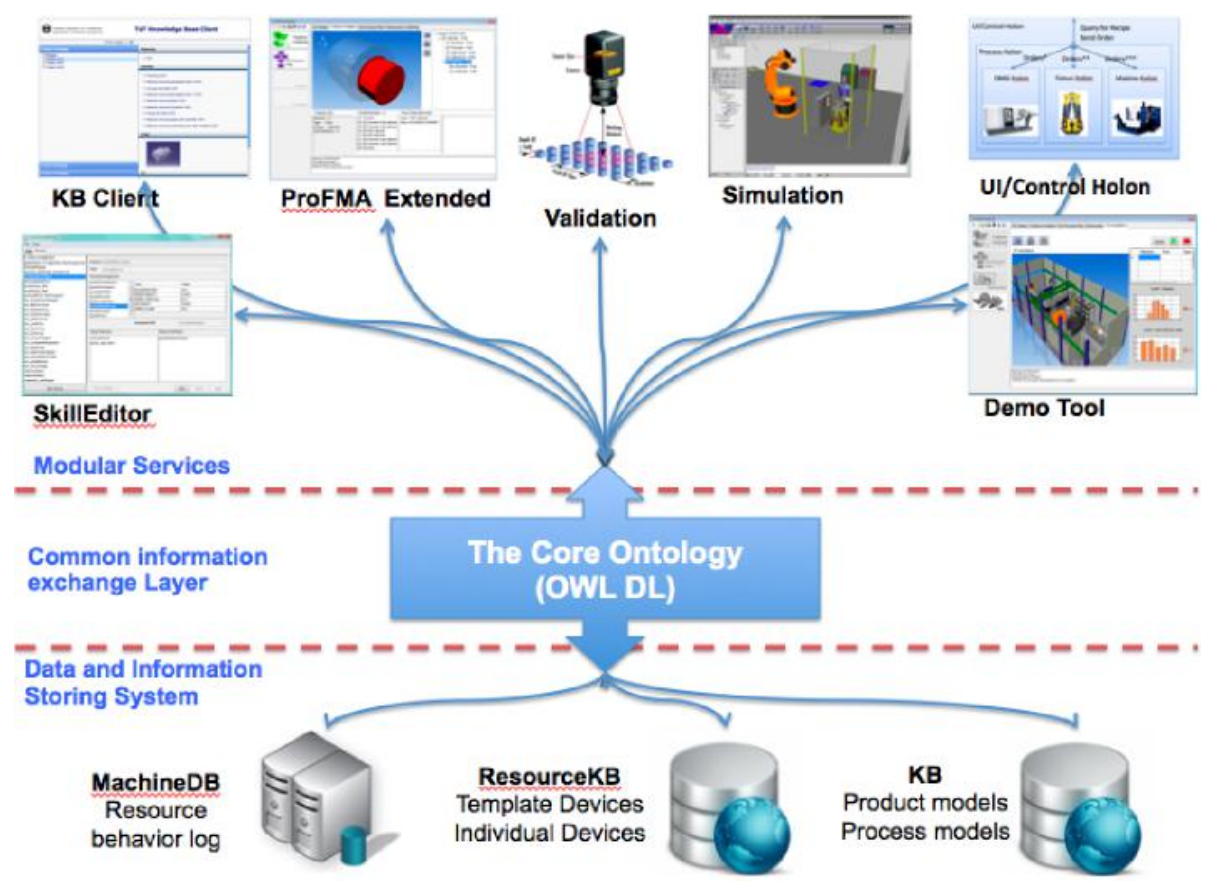

Fig. 1. Modular ICT architecture for HMS 
Another option is a common knowledge model. Standards and information models inside commercial systems try to enforce one representation that would fit all fields, however, their approach does not support extendable and open representations. In this approach design and control system is as open as possible, since the knowledge representation is formed in such way that it supports extensions and scalability of its form. The approach do force the use of common information model as illustrated in Figure 1, for the share of information, not creation of it.

The architecture is divided into three separate layers: databases, formal semantic operation logic (the knowledge representation) and services that utilize the commonly available information. Once the storing method is extracted from the logic and services, the new concepts can emerge. By dividing the data reserves, operation logic and services into separate layers connected with interfaces the upgrading of layers becomes independent of each others. This allows services to be extended, replaced and modified through out of their own life-cycles without disrupting the validity and consistency of the core domains. The modular approach in ICT supports the need of adaptive production system, since the services can be added or removed based upon a need while proposing minimum set of disturbances to the whole system.

It is seen also that the small and medium sized vendors can make new business openings, instead of modifying highly tailored and closed solutions used today. When new services are not implemented inside a legacy system, but into open design environment as additional modules, these can be marketed to wider industry sector. Modular ICT architecture can be also upgraded section by section depending on the life-cycle of each services and how these comply to the life-cycle of overall business trend of the manufacturing company. This is seen also as an asset for achieving interactive virtual collaboration environment.

\subsection{Service View}

The service view is dedicated to different queries, knowledge reasoning and knowledge processing. The architecture is designed in such way that each of the modules can be replaced with a new module or removed if needed. The connection of the modules is based on the shared information model such as described in detail in $[8,10]$. The services in the environment are designed by keeping the modularization principles in mind. Each of the tools are contributing their specific information to the common information model. The tools provide one or two main fucntionalities to the software environment. The tools do not have specific interfaces with eachothers, only via information model. The modular design of the software allows changes to be applied to the tools with minimum disturbances. Each of these tools requires specific domain related information and by processing the information they provide a set of services.

\subsection{Common Information Exchange Layer}

The common information model provides formal and thus computer interpretable knowledge representation for product, process and resource information. The product information is represented as geometrical and non-geometrical features. The resource information describes the capabilities of each resource element inserted into the system. Since resources are products as well, these can be visualized and represented 
with geometerical and non-geomterical features. Processes in this environment are desribed only with order and time spent. The information model, operation logic, is designed to be extended if and when needed. There are no limits for extending the knowledge representation while allowing the old services (software) to function. The operation logic in this case was formalized OWL DL (Web Ontology Language with Description Logic) representation $[9,10]$. Even though it is understood that OWL DL do restrict the representation as well as reasoning procedures, in this implementation it is seen sufficient. The operation logic resides in the developed Knowledge Base (KB) [9]. The approach differs from previous attempts such described in [1], [11],[12] and [13] by utilising the ontology as information architecture and information sharepoint instead of generating strict rules for actual information processing. In a sense this knowledge description is static while services are dynamic.

\subsection{Data Storage}

The data storing is extracted from the operation logic. The collected production values are stored, filtered and prepared in several distinct databases. The prepared production parameters are saved into or referred from the KB. Similarly the additional, and perhaps closed models, such as simulation files or CAD files are referred via KB.

\section{$4 \quad$ Laboratory Implementation}

The modular ICT concept was tested with three production scenarios. The first scenario was a part's feature manufacturing, such as drilling a hole. Second case was ordering a complete part and third scenario was a assembly of a three piece product. The system in control level does not distinguish the tasks needed to be done, the tasks are purely assigned based on the capability requirements.

\subsection{Holonic Manufacturing System}

Agent-based and holonic systems take more dynamic approach to cope with the changeability requirements. The HMS concept implemented here views the manufacturing system as one entity consisting of autonomous modules (holons) with distributed control. The holons act as autonomous entities able to fulfill their own goals, as well as communicate with other holons and form set of holons, holarchies, through common interfaces and negotiation process. This operation environment differs from the agent-based autonomous systems, such as Evolving Production System (EPS) from [12], since there is human-machine cooperation involved. The goal has been to attain the benefits that holonic organization provides to living organisms and societies, in manufacturing, i.e., stability in the face of disturbances, adaptivity and flexibility in the face of change, and efficient use of available resources through self-organization ability [7, 10].

The HMS paradigm addresses the agile reaction to disturbances at the shop floor level in volatile environments and it is built upon a set of autonomous and cooperative holons, each one being a representation of a manufacturing component, i.e., a physical 
resource such as a robot, $\mathrm{CNC}$ centre and conveyor, or a logical entity, such as orders. The main character of the built holonic system is that the status of the production system and desired goal (defined as order connected to product model) are known, but the steps for reaching the goal, in this case the routing order of the parts in the factory floor, is not predefined [10].

\subsection{Implemented Operation Principles for HMS}

The holonic system follows service oriented architecture (SOA), where the resources provide services through their capabilities. The holons organize themselves in a hierarchical manner. This hierarchy is called holarchy. The upper owner holon is responsible for arranging the holons in such a way that the desired task can be accomplished. The system is robust in a way that new holons and holarchies can join and leave the system without disturbing the whole system.

In this concept the both hardware and software modules are service providers. When an order enters to the holonic framework, the system will search for those resources, which can alone, or with some other resource, satisfy the requested service. The holons will then negotiate to determine the best resource for the given situation or the part is directed to first available resource combination that has the capability to produce the part or specific feature [8]. The reasoning is done based on mapping the geometrical and non-geometrical features of a part/product to the resource characteristics and determining possible matches.

In the presented implementation the holons are running processes on an operating system and communication between the holons is done by using normal TCP/IP transport protocol. Holons communicate in a purely hierarchical way, which means that every holon creates single communication connection to the host holon. Host holon acts as an actual implementation of a specific solution as well as a message broker that relays the messages between holons connected to it. The messages are encoded using BEncode serialization method, which is a lightweight, semi-humanreadable serialization format $[8,10]$.

\subsection{Core of the Modular ICT}

In this case study the whole system architecture has several different interoperating software modules each providing one or two essential functions for the whole holonic manufacturing system. For example the holon UI, which controls the actual production can be replaced with a commercialtool that provides queuing functionality for the system. The core services of the modular architecture are:

- Content creation: Pro-FMA [5] is used to define the product requirements from the product model given in VRML or X3D format. Pro-FMA recreates the features lost in translation and formalizes the representation according to the defined ontology. Visualization is provided with X3D and actual processed information is in OWL DL/RDF format.

- Context creation: Skill Editor allows user to add devices to the ontology and assign them capabilities and capability parameters and enables creating associations between the capabilities, in other words creating rules about 
which simple capabilities are needed to form combined capabilities [7]. The device descriptions can range from capability definitions to feature-level representations.

- Verification: A simulation tool is used for creating the manufacturing or assembly scenarios. Since the environment is holonic by nature, it is accepted that the simulation only expresses possible solutions. The operation principles inside the simulation follows also the holonic guidelines. This means that part or product is routed to first available and capable cell or station.

- Ordering: Decision Making and Ordering Tool (DeMO tool) is used for setting up the orders in this environment. The tool supports the viewing of the simulation as its minor function. The main function of the DeMO tool is to verify connection to factory floor and forward the orders to the Control Holon.

- $\quad$ Operations Management: The process flow and distribution of tasks to each manufacturing or assembly cell is done with the Control Holon. This system distributes in current implementation the tasks to suitable and available cells or stations based on the capability requirements defined by Pro-FMA earlier. The status of the available resources and capabilities is monitored constantly.

- Common Knowledge Representation: The KB and ResourceKB store the information created by Pro-FMA, Skill Editor and DeMO tool. This system serves also as reference architecture, since it can handle closed models as references. Simulation model can be attached to product definition if needed. Similarly closed sub-programs and CAx models can be associated with the part/product/resource description.

- Content Verification: A web-based KB client is used for human friendly information browsing. This tool serves as online product data management (PDM) user interface (UI).

The additional SW services the system can have upon a need are:

- $\quad$ Traditional Operations Management: The operation management module can be re-placed on the fly with a commercial software. The system is thus transformed into traditional production system, where a human operator is in control of the process orchestration.

- $\quad$ Machine Vision based Validation: The system can have validation option in a form of camera set up. In this case the validation and verification with a camera is used for verifying that the laser processes are producing needed quality. At the moment the implementation of material adding laser with machine vision quality control is on the way.

\section{Conclusions}

This research approach introduced a modular ICT concept for supporting dynamically evolving assembly and manufacturing system. The novelty of this approach is that the services are fully modular and can be updated/replaced/removed without disturbing the system. New services and new information can be added during the life-cycle of 
the business itself. This approach differs from the developed academy and commercial tools by

1) utilizing the part's and product's geometrical and non-geometrical features (referred as content) as real input information for the automatic reasoning processes, and

2) the architecture does not force all SW tools to be developed within one strict framework, only to use common information model for sharing the content and context information.

The approach is seen also to enhance the virtual collaboration between service providers, since there is no restriction forced from other systems to develop new services. The ultimate goals in this particular research effort were to provide such information architecture that allows different utilization of domain knowledge, while keeping the core information consistent and valid throughout the life-cycles of that particular set of information.

According to Chavalarias et al.[2], there is no doubt that one of the main characteristics of complex and adaptive production platforms in the future will be the everincreasing utilization of ICT (information and communication tech- nology). However, while the industrial world has seen the possible advantages, the implementations fall short as a result of the changes to the whole production paradigm that are required, going from pre-planned hierarchical systems to adaptive and selforganizing complex systems [2], [3].

Authors are confident that in future the trend is towards a service oriented design and production environment, where one single large and closed system cannot fulfill the needs of the system's adaptability.

Acknowledgments. The authors would like to thank Tekes, Finland's National Funding Agency, KIPPcolla and CSM projects and projects' consortia for support and feedback during the research.

\section{References}

1. Borgo, S., Leit, P.: Foundations for a core ontology of manufacturing. Integrated Series in Information Systems, vol. 14 (2007)

2. Chavalarias, D., Cardelli, L., Kasti, J., et al.: Complex Systems: Challenges and opportunities, an orientation paper for complex systems research in fp7, European Commission (2006)

3. Cotsaftis, M.: A passage to complex systems. In: Bertelle, C., Duchamp, G.H.E., KadriDahmani, H. (eds.) Complex Systems and Self-organization Modeling. Springer, Heidelberg (2009)

4. Dept. of Homeland Security (DoHS), NAT, Cyber Security Division: Catalog of Control Systems Security, Recommendations for Standards Developers (2008)

5. Garcia, F., Lanz, M., Luostarinen, P., Tuokko, R.: Process planning based on feature recognition methods. In: International Symposium of Assembly and Manufacturing (ISAM), Tampere, Finland, May 25-27. IEEE Press (2011) 
6. Kim, J., Peng, Y., Ivezic, N., Shin, J.: An Optimization Approach for Semantic- based XML Schema Matching. International Journal of Trade, Economics and Finance 2(1), 78-86 (2011)

7. Jarvenpaa, E., Luostarinen, P., Garcia, F., Lanz, M., Tuokko, R.: Dynamic Operation Environment - Towards Intelligent Adaptive Production Systems. In: International Symposium of Assembly and Manufacturing (ISAM), Tampere, Finland, May 25-27. IEEE Press (2011)

8. Jarvenpaa, E., Luostarinen, P., Lanz, M., Tuokko, R.: Presenting capabilities of resources and resource combinations to support production system adaptation. In: International Symposium of Assembly and Manufacturing (ISAM), Tampere, Finland, May 25-27. IEEE Press (2011)

9. Lanz, M.: Logical and Semantic Foundations of Knowledge Representation for Assembly and Manufacturing Processes, PhD thesis, Tampere University of Technology (2010)

10. Lanz, M., Jarvenpaa, E., Luostarinen, P., Tenhunen, A., Tuokko, R., Rodriguez, R.: Formalizing Connections between Products, Processes and Resources - Towards Semantic Knowledge Management System. In: Proceedings of Swedish Production Symposium (SPS 2011), Sweden (2011)

11. Lohse, N.: Towards an Ontology Framework for the Integrated Design of Modular Assembly Systems, PhD thesis, University of Nottingham (2006)

12. Onori, M.: Evolvable Assembly System - A new paradigm? In: Proceedings of the 33rd International Symposium on Robotics, Stockholm, Sweden (2002)

13. FP6 Pabadis'Promise: D3.1 Development of manufacturing ontology, project deliverable, The PABADIS'PROMISE consortium (2006)

14. Ribeiro, L., Barata, J., Pimentaõ, J.: Where Evolvable Production Systems meet Complexity Science. In: International Symposium of Assembly and Manufacturing (ISAM), Tampere, Finland, May 25-27. IEEE Press (2011) 